COMMUNICATIONS IN

ANALYSIS AND GEOMETRY

Volume 12, Number 3, 601-630, 2004

\title{
$K$ Energy and $K$ Stability on Hypersurfaces
}

\section{ZHIQIN LU}

\section{Introduction.}

Suppose that $M$ is a compact Fano manifold. That is, $M$ is a compact Kähler manifold with positive first Chern class. One of the most important problems in Kähler geometry is the existence of Kähler metrics of constant scalar curvature. It is believed that the problem is related to certain notion of stability in the sense of Geometric Invariant Theory.

In Tian [17] and Donaldson [4], the notion of $K$ stability was introduced. In the first three sections of this paper, we use the notations in [17] to derive our theorems. In the last section, we discuss the definition of [4] and some observations motivated by that paper.

Let $M$ be a Fano manifold that is embedded in $C P^{n}$ by the $k$-th power of the anticanonical line bundle, where $k$ is a positive integer. Let $\sigma(t)$ be a one parameter family of automorphisms of $C P^{n}$. We write

$$
\sigma(t)\left[Z_{0}, \cdots, Z_{n}\right]=\left[t^{\lambda_{0}} Z_{0}, \cdots, t^{\lambda_{n}} Z_{n}\right]
$$

for integers $\lambda_{0}, \cdots, \lambda_{n}$ with $\sum \lambda_{i}=0$. Then we can define a family of metrics $\omega_{t}=\sigma(t)^{*} \omega_{F S}$ on $M$ such that $\alpha \omega_{t} \in c_{1}(M)$, where $\alpha$ is a rational number. Let $\mathcal{M}\left(\omega, \omega_{t}\right)$ be the $K$ energy with respect to the metric $\alpha \omega$ and $\alpha \omega_{t}$ (for the definition of the $K$ energy, see next section). It is known that

$$
\lim _{t \rightarrow 0} t \frac{d}{d t} \mathcal{M}\left(\omega, \omega_{t}\right)=A
$$

exists [17]. If $\mathcal{M}\left(\omega, \omega_{t}\right)$ has a lower bound, then $A \leq 0$. Since the one parameter family of automorphisms $\sigma(t)$ is generated by the holomorphic vector field $X=\sum \lambda_{i} Z_{i} \frac{\partial}{\partial Z_{i}}$, we come up with the following definition [17]:

\footnotetext{
${ }^{1}$ Supported by the NSF grant DMS 0204667 and the Alfred P. Sloan Research Fellowship.
} 
Definition 1.1. We say that $M$ is $K$ stable if for any holomorphic vector field $X$ on $C P^{n}$ with $\lambda_{0}, \cdots, \lambda_{n}$ integers and $\lambda_{0}^{2}+\cdots+\lambda_{n}^{2} \neq 0$,

$$
\lim _{t \rightarrow 0} t \frac{d}{d t} \mathcal{M}\left(\omega, \omega_{t}\right)<0 .
$$

If the above quantity is nonpositive for all vectors $X$ on $C P^{n}$, we say $M$ is $K$ semistable.

The general setting which relates the $K$ energy and the Futaki invariant (in the case of hypersurface) is as follows: Let $M$ be a hypersurface of $C P^{n}$. Let $X$ be a vector field of $C P^{n}$. Suppose $M$ is defined by a polynomial $F=0$ and let $F_{t}=\sigma(-t)^{*} F$. The degeneration of $M$ by $X$ is defined as the hypersurface in $\mathbb{C} \times C P^{n}$ by $G(t, Z)=F_{t}(Z)=0$. The central fiber of the degeneration is defined as the intersection of the degeneration with the set $\{0\} \times C P^{n}$, excluding the factor $t=0$.

In [2] or [17], the quantity $A$ in (1.1) is represented as the (real part) of the (generalized) Futaki invariant of the central fiber if the central fiber is a normal variety. It is not hard to see that the exact same proof can go through if we assume that the central fiber does not have multiplicity greater than 1. In particular, we can define the Futaki invariant on algebraic cycles with multiplicity 1 pretty much the same way as in the smooth case.

Remark 1.1. For the sake of simplicity, we don't distinguish the notations of $K$ stability and $K$ semi-stability in this paper. $K$ stable in this paper means either $K$ stable or $K$ semi-stable. On the other side, for the applications in Geometric Invariant Theory, we just need to assume that $t$ is a real number and $\lambda_{0}, \cdots, \lambda_{n}$ are rational numbers, although the main idea of this paper extends to the case where $t, \lambda_{0}, \cdots, \lambda_{n}$ are complex numbers.

The motivation of our work is to find an effective way to verify the $K$ stability for hypersurfaces. In general, this is a harder problem than the problem of finding an effective way to compute the Futaki invariant, because the $K$ energy is the nonlinear version of the "Futaki" invariant(see [10]). By the work of [2] or [17], if the central fiber is normal, the quantity $A$ is the real part of the corresponding Futaki invariant. However, the technical difficulty in the proof is that the degeneration of a hypersurface under a one parameter subgroup is "generically" an algebraic cycle of multiplication greater than 1. If that is the case, we would not be able to generalize the argument in [2] directly. In fact, our result shows that the limit may not depend on the central fiber alone. To see this, we consider the "generic" case where the central fiber is represented by the algebraic cycle $Z_{0}^{i_{0}} \cdots Z_{n}^{i_{n}}=0$. All 
the information of the central fiber is contained in the vector $\left(i_{0}, \cdots, i_{n}\right)$. However, (1.6) shows that we have the other term

$$
\sum_{i=0}^{n} \int_{0}^{\infty} \varphi_{i}^{\prime}(x)\left(\varphi_{i}^{\prime}(x)-1\right) d x
$$

that can't be recovered from the central fiber. Thus the left hand side of (1.6) depends not only on the central fiber, but also on the whole degeneration $F_{t}$.

In this paper, we overcome the above difficulty in the case that the central fiber is of multiplicity greater than one. We first represent the $K$ energy into an explicitly formula(Theorem 2.5). Then we carefully analyze the integrand in the formula by using some analytic techniques and a recent result of Phong and Sturm [14] to get the conclusion.

This paper can be viewed as a nonlinearized version of the paper [5] of the author, where the Futaki invariant of a hypersurface of $C P^{n}$ was computed.

Phong and Sturm studied $K$ stability for arbitrary smooth manifolds in $C P^{N}$ [13]. They also studied the linearized version, i.e., the computation of the Futaki invariant, in [15]. In order to establish the result in the general case, they make use of the Chow point and Deligne pair. The Chow point, which is a hypersurface of some Grassmannian, contains all the information of the original manifold.

The Chow stability (which is closely related to $K$ stability) was studied by Paul [11] and more recently by Paul and Tian [12], where they have formulated the stability in terms of double Chow points.

Before stating the main result, we setup notations: let $M$ be defined by the zeros of the polynomial

$$
F=\sum_{i=0}^{p} a_{i} Z_{0}^{\alpha_{0}^{i}} \cdots Z_{n}^{\alpha_{n}^{i}}
$$

of degree $d$. Let $\left(\lambda_{0}, \cdots, \lambda_{n}\right)$ be rational numbers satisfying $\sum \lambda_{i}=0$. Let

$$
\lambda=\operatorname{Max}_{0 \leq i \leq p}\left(\sum_{k=0}^{n} \lambda_{k} \alpha_{k}^{i}\right) .
$$

Let

$$
\varphi\left(x_{0}, \cdots, x_{n}\right)=\operatorname{Min}_{0 \leq i \leq p}\left(-\sum_{k=0}^{n} \lambda_{k} \alpha_{k}^{i}+\sum_{k=0}^{n} \alpha_{k}^{i} x_{k}\right)
$$

and let

$$
\varphi_{i}(x)=\varphi(0, \cdots, \underset{i}{x}, \cdots, 0)
$$


Then we have the following

Theorem 1.1. For "generic" (See section 3 for details) $\left(\lambda_{0}, \cdots, \lambda_{n}\right)$, we have

$$
\begin{aligned}
& \lim _{r \rightarrow 0} t \frac{d}{d t} \mathcal{M}(t) \\
= & \frac{2}{d}\left(-\frac{\lambda(d-1)(n+1)}{n}+\sum_{i=0}^{n} \int_{0}^{\infty} \varphi_{i}^{\prime}(x)\left(\varphi_{i}^{\prime}(x)-1\right) d x\right) .
\end{aligned}
$$

Since for a Kähler-Einstein manifold, the $K$ energy has a lower bound, we have the following:

Theorem 1.2. If $M$ is a Kähler-Einstein hypersurface with positive first Chern class, then we have

$$
-\frac{\lambda(d-1)(n+1)}{n}+\sum_{i=0}^{n} \int_{0}^{\infty} \varphi_{i}^{\prime}(x)\left(\varphi_{i}^{\prime}(x)-1\right) d x \leq 0
$$

for any $\lambda_{0} \cdots, \lambda_{n} \in \mathbb{R}$ with $\sum \lambda_{i}=0$.

Proof of Theorem 1.2. The expression in the theorem is continuous and homogeneous with respect to $\lambda_{0}, \cdots, \lambda_{n}$. So by taking the limit, we proved that the inequality is valid for any choice of $\lambda_{0}, \cdots, \lambda_{n} \in \mathbb{R}$.

This paper is a refinement of the paper [7]. We rewrite the introduction of this paper in order to cite the important papers of Donaldson [4], PhongSturm $[13,15]$. We also give some new observations in the last section motivated by the work [4].

Acknowledgment. The author thanks P. Li, D.H. Phong and G. Tian for the encouragement during the preparation of this paper. Special thanks to L. Katzarkov who helped the author clarify a lot of concepts in algebraic geometry. The author had discussions on this topic with D. H. Phong and J. Sturm during his visit to Le Centre de Recherches Mathématiques at Université de Montréal in summer, 2003. Their thoughts influence the current version of this paper. Finally, the author thanks the referee for the careful reading of this very technical paper.

\section{An explicit formula for the $K$ energy.}

In this section, we give an explicit formula for the $K$ energy of smooth hypersurfaces of $C P^{n}$. 
First, let's recall the definition of the $K$ energy [10]. Let $M$ be a compact Kähler manifold with positive first Chern class $c_{1}(M)$. Let $\omega_{0}, \omega_{1} \in c_{1}(M)$ and let $\omega_{1}=\omega_{0}+\frac{\sqrt{-1}}{2 \pi} \partial \bar{\partial} \xi$ for a smooth function $\xi$. We put $\omega_{s}=\omega_{0}+$ $s \frac{\sqrt{-1}}{2 \pi} \partial \bar{\partial} \xi$ and define

$$
\mathcal{M}\left(\omega_{0}, \omega_{1}\right)=-\frac{1}{V} \int_{0}^{1}\left(\int_{X} \xi\left(R\left(\omega_{s}\right)-m\right) \omega_{s}^{m}\right) d s
$$

where $R\left(\omega_{s}\right)$ is the scalar curvature of the metric, $m$ is the complex dimension of $M$, and $V$ is the volume of $X$ with respect to $\omega_{0}$. The functional $\mathcal{M}$, which is called the $K$ energy by Mabuchi, has the properties:

Proposition 2.1. Using the notations as above, we have

1. $\mathcal{M}\left(\omega_{0}, \omega_{1}\right)=-\mathcal{M}\left(\omega_{1}, \omega_{0}\right)$,

2. $\mathcal{M}\left(\omega_{0}, \omega_{1}\right)+\mathcal{M}\left(\omega_{1}, \omega_{2}\right)=\mathcal{M}\left(\omega_{0}, \omega_{2}\right)$,

where $\omega_{0}, \omega_{1}, \omega_{2} \in c_{1}(X)$, and are Kähler metrics.

From now on, let's assume that $\omega$ is the Kähler form of the Fubini-Study metric of $C P^{n}$. Let $M$ be a hypersurface in $C P^{n}$ defined by the polynomial $F=0$ of degree $d$. Of course, we need $d \leq n$ to insure that $M$ is Fano. Let $\lambda_{0}, \cdots, \lambda_{n}$ be integers such that $\sum_{i=0}^{n} \lambda_{i}=0$. Let $F_{t}$ be the polynomial defined by

$$
F_{t}\left(Z_{0}, \cdots, Z_{n}\right)=F\left(t^{-\lambda_{0}} Z_{0}, \cdots, t^{-\lambda_{n}} Z_{n}\right),
$$

and let $M_{t}$ be the hypersurface defined by the zero set of $F_{t}$. Geometrically, $M_{t}$ is the image of $M$ under the automorphism $\sigma(t)$ generated by the holomorphic vector field $X=\sum_{i=0}^{n} \lambda_{i} Z_{i} \frac{\partial}{\partial Z_{i}}$. The automorphisms $\sigma(t)$ can be written as $\sigma(t)\left(\left[Z_{0}, \cdots, Z_{n}\right]\right)=\left[t^{\lambda_{0}} Z_{0}, \cdots, t^{\lambda_{n}} Z_{n}\right]$. Using these automorphisms, one can define a family of Kähler forms $\omega_{t}=\sigma(t)^{*} \omega$ on $M$. It is easy to see that both $(n-d+1) \omega$ and $(n-d+1) \omega_{t}$ are Kähler forms of $M$ in the cohomological class $c_{1}(M)$. Define $\mathcal{M}(t)=\mathcal{M}\left((n-d+1) \omega,(n-d+1) \omega_{t}\right)$. It is a well known result [10] that if $M$ admits a Kähler-Einstein metric, then $\mathcal{M}(t)$ has a lower bound.

Proposition 2.2. Using the notations as above, we have

$$
t \frac{d}{d t} \mathcal{M}(t)=\frac{2(n-1)}{d} \int_{M_{t}}\left(\operatorname{Ric}\left(\left.\omega\right|_{M_{t}}\right)-\left.(n-d+1) \omega\right|_{M_{t}}\right) \theta \omega^{n-2}
$$


where $\theta$ is defined as

$$
\theta=-\frac{\sum_{i=0}^{n} \lambda_{i}\left|Z_{i}\right|^{2}}{\sum_{i=0}^{n}\left|Z_{i}\right|^{2}}
$$

and $\operatorname{Ric}\left(\left.\omega\right|_{M_{t}}\right)$ is the Ricci form of $\left.\omega\right|_{M_{t}}$.

Proof. It basically follows from Proposition 2.1. Also see [2, Lemma 2.1] for details.

The following lemma can be found in [16], we include the proof here for the sake of completeness.

Lemma 2.1. Let $M$ be the smooth hypersurface defined as the zero of $\{F=$ $0\}$. We use $\omega$ to denote the Fubini-Study metric on $C P^{n}$ as well as the Kähler form on $M$, which is the restriction of $\omega$ on $M$. Let

$$
\xi=\log \frac{|\nabla F|^{2}}{\left(\sum_{i=0}^{n}\left|Z_{i}\right|^{2}\right)^{(d-1)}},
$$

where $\left[Z_{0}, \cdots, Z_{n}\right]$ is the homogeneous coordinate in $C P^{n}$. Then we have

$$
\operatorname{Ric}(\omega)-(n-d+1) \omega=-\frac{\sqrt{-1}}{2 \pi} \partial \bar{\partial} \xi .
$$

Proof. Without losing generality, we prove the above lemma on the open set

$$
U_{0}=\left\{\left[Z_{0}, \cdots, Z_{n}\right]|| Z_{0}\left|>\frac{1}{2}\right| Z_{j} \mid, j=1, \cdots, n\right\}
$$

in $C P^{n}$. The local coordinate system on $U_{0}$ is $z=\left(z_{1}, \cdots, z_{n}\right)$ where $z_{i}=$ $Z_{i} / Z_{0}$ for $i=1, \cdots n$. Under this coordinate system, the Fubini-Study metric can be written as

$$
\omega=\frac{\sqrt{-1}}{2 \pi} g_{i j} d z_{i} \wedge d \bar{z}_{j}=\frac{\sqrt{-1}}{2 \pi} \sum_{i, j=1}^{n}\left(\frac{\delta_{i j}}{1+|z|^{2}}-\frac{z_{j} \bar{z}_{i}}{\left(1+|z|^{2}\right)^{2}}\right) d z_{i} \wedge d \bar{z}_{j},
$$

where $|z|^{2}=\sum\left|z_{i}\right|^{2}$. Let's further assume that in a small open set $V$ of $U_{0}$, from the equation $F=0$, we can solve $z_{1}$. Namely,

$$
z_{1}=z_{1}\left(z_{2}, \cdots, z_{n}\right)
$$

for a holomorphic function $z_{1}$. Let the Kähler form $\omega$ on $V$, under the local coordinate system $\left(z_{2}, \cdots, z_{n}\right)$, be written as

$$
\omega=\frac{\sqrt{-1}}{2 \pi} \sum_{i, j=2}^{n} \tilde{g}_{i \bar{j}} d z_{i} \wedge d \bar{z}_{j}
$$


and let $a_{i}=\frac{\partial z_{1}}{\partial z_{i}}, i=2, \cdots, n$. Then by (2.5) and (2.6), we have

$$
\begin{aligned}
\tilde{g}_{i \bar{j}} & =\frac{\delta_{i j}}{1+|z|^{2}}-\frac{z_{j} \bar{z}_{i}}{\left(1+|z|^{2}\right)^{2}}-\frac{z_{j} \overline{z_{1}} a_{i}}{\left(1+|z|^{2}\right)^{2}}-\frac{z_{1} \bar{z}_{i} \bar{a}_{j}}{\left(1+|z|^{2}\right)^{2}} \\
& +\frac{a_{i} \bar{a}_{j}}{1+|z|^{2}}-\frac{\left|z_{1}\right|^{2} a_{i} \bar{a}_{j}}{\left(1+|z|^{2}\right)^{2}}
\end{aligned}
$$

for $i, j=2, \cdots, n$. We want to compute the $\operatorname{determinant} \operatorname{det}\left(\tilde{g}_{i \bar{j}}\right)$. In order to do this, we let

$$
K_{i \bar{j}}=\delta_{i j}+a_{i} \bar{a}_{j}-\frac{1}{1+|z|^{2}}\left(\bar{z}_{i}+\bar{z}_{1} a_{i}\right) \overline{\left(\bar{z}_{j}+\bar{z}_{1} a_{j}\right)}
$$

Then

$$
\tilde{g}_{i \bar{j}}=\frac{1}{1+|z|^{2}} K_{i \bar{j}}, \quad i, j=2, \cdots, n
$$

Let

$$
\begin{aligned}
& A=\left(a_{2}, \cdots, a_{n}\right) \\
& B=\left(\bar{z}_{2}+\bar{z}_{1} a_{2}, \cdots, \bar{z}_{n}+\bar{z}_{1} a_{n}\right) .
\end{aligned}
$$

Then the matrix $K=\left(K_{i \bar{j}}\right)$ can be represented by

$$
K=I+A^{T} \bar{A}-\frac{1}{1+|z|^{2}} B^{T} \bar{B}
$$

A straightforward computation gives

$$
\begin{aligned}
& K A^{T}=\left(1+|a|^{2}\right) A^{T}-\frac{1}{1+|z|^{2}}\left(\bar{B} A^{T}\right) B^{T} ; \\
& K B^{T}=\left(\bar{A} B^{T}\right) A^{T}+\left(1-\frac{|B|^{2}}{1+|z|^{2}}\right) B^{T} .
\end{aligned}
$$

Thus the vector space spanned by the vectors $A, B$ is $K$-invariant. Furthermore, on the complement of the vector space, $K$ is the identity. So we have

$$
\begin{aligned}
& \operatorname{det} K=\left(1+|a|^{2}\right)\left(1-\frac{|B|^{2}}{1+|z|^{2}}\right)+\frac{1}{1+|z|^{2}}\left|\bar{B} A^{T}\right|^{2} \\
& =\frac{1}{1+|z|^{2}}\left(1+|a|^{2}+\left|\sum_{i=2}^{n} a_{i} z_{i}-z_{1}\right|^{2}\right) .
\end{aligned}
$$


Let $f$ be the defining function of $M$ on $U_{0}$, i.e.

$$
f=F\left(1, \frac{Z_{1}}{Z_{0}}, \cdots, \frac{Z_{n}}{Z_{0}}\right)=\frac{F}{Z_{0}^{d}}
$$

Then

$$
\frac{\partial z_{1}}{\partial z_{k}}=-\frac{\frac{\partial f}{\partial z_{k}}}{\frac{\partial f}{\partial z_{1}}}=-\frac{F_{k}}{F_{1}}, \quad(k=2, \cdots, n)
$$

where we define $F_{k}=\frac{\partial F}{\partial Z_{k}}$ for $k=0, \cdots, n$. Thus by the homogeneity of $F$, we have

$$
\begin{aligned}
& \left(\sum_{i=2}^{n} a_{i} z_{i}\right)-z_{1}=-\left(\sum_{i=2}^{n} \frac{Z_{i}}{Z_{0}} \frac{F_{i}}{F_{1}}\right)-\frac{Z_{1}}{Z_{0}} \\
& =-\frac{1}{Z_{0} F_{1}}\left(\sum_{i=1}^{n} Z_{i} F_{i}\right)=\frac{F_{0}}{F_{1}}
\end{aligned}
$$

on $M$. Using (2.7), (2.8), and (2.10), we have

$$
\operatorname{det} \tilde{g}_{i \bar{j}}=\frac{1}{\left(1+|z|^{2}\right)^{n}} \frac{1}{\left|F_{1}\right|^{2}}\left(\sum_{k=0}^{n}\left|F_{k}\right|^{2}\right) .
$$

Then by $(2.3)$

$$
\operatorname{det} \tilde{g}_{i \bar{j}}=\frac{1}{\left(1+|z|^{2}\right)^{n-d+1}} \cdot \frac{1}{\left|\frac{\partial f}{\partial z_{1}}\right|^{2}} \cdot e^{\xi} .
$$

(2.4) follows from the formula of the Ricci curvature and the above equation.

In order to represent the $K$ energy in terms of the polynomial $F$, we need the following purely algebraic lemma:

Lemma 2.2. With the same notations as above, let $\eta$ be a $(1,1)$ form on $C P^{n}$. Let $\pi: C^{n+1} \rightarrow C P^{n}$ be the projection. Let

$$
\pi^{*} \eta=\frac{\sqrt{-1}}{2 \pi} \sum_{i, j=0}^{n} \tilde{a}_{i \bar{j}} d Z_{i} \wedge d \bar{Z}_{j}
$$

Then on $M$,

$$
\eta \wedge \omega^{n-2}=\frac{|Z|^{2}}{n-1}\left(\sum_{i=0}^{n} \tilde{a}_{i \bar{i}}-\frac{\sum_{i, j=0}^{n} \tilde{a}_{i \bar{j}} F_{j} \bar{F}_{i}}{|\nabla F|^{2}}\right) \omega^{n-1}
$$

for $|Z|^{2}=\sum_{i=0}^{n}\left|Z_{i}\right|^{2}$. 
Remark 2.1. The righthand side of (2.13) is well defined because $\tilde{a}_{i \bar{j}}$ for $i, j=0, \cdots, n$ are homogeneous functions of order $(-2)$.

Proof. As in the proof of the previous lemma, we can consider the problem only on $U_{0} \cap\left\{\frac{\partial F}{\partial Z_{1}} \neq 0\right\}$, without losing generality. Define $A_{i \bar{j}}$ on $C P^{n}$ as follows:

$$
\begin{aligned}
& \eta \wedge \omega^{n-2}=\left(\frac{\sqrt{-1}}{2 \pi}\right)^{n-1}(-1)^{\frac{1}{2}(n-1)(n-2)} \\
& \cdot \sum_{i, j=1}^{n}(-1)^{i+j} A_{i \bar{j}} d z_{1} \wedge \cdots \wedge \hat{d z_{i}} \cdots \wedge d z_{n} \wedge d \bar{z}_{1} \wedge \cdots \wedge d \hat{\bar{z}}_{j} \cdots \wedge d \bar{z}_{n},
\end{aligned}
$$

where the "hat' symbol " $\wedge$ " over $d z_{j}$ and $d \bar{z}_{j}$ means these two differential forms are deleted from the expression. Define $b=\left(b_{1}, \cdots, b_{n}\right)$ by

$$
b=\left(1,-a_{2}, \cdots,-a_{n}\right)=\left(1,-\frac{\partial z_{1}}{\partial z_{2}}, \cdots,-\frac{\partial z_{1}}{\partial z_{n}}\right)=\left(1, \frac{F_{2}}{F_{1}}, \cdots, \frac{F_{n}}{F_{1}}\right) .
$$

Then by (2.14), we have

$$
\begin{aligned}
& \eta \wedge \omega^{n-2}=\left(\frac{\sqrt{-1}}{2 \pi}\right)^{n-1}(-1)^{\frac{1}{2}(n-1)(n-2)} \\
& \cdot \sum_{i, j=1}^{n} A_{i \bar{j}} b_{i} \bar{b}_{j} d z_{2} \wedge \cdots \wedge d z^{n} \wedge d \bar{z}^{2} \wedge \cdots \wedge d \bar{z}^{n}
\end{aligned}
$$

on $M$. Thus in order to prove (2.13), we just need to compute $\sum A_{i \bar{j}} b_{i} \bar{b}_{j}$. To this end, let

$$
\eta=\frac{\sqrt{-1}}{2 \pi} \sum_{k, l=1}^{n} a_{k \bar{l}} d z_{k} \wedge d \bar{z}_{l},
$$

and fix $r, s$. By (2.14), we have

$$
\begin{aligned}
& \frac{\sqrt{-1}}{2 \pi} d z_{r} \wedge d \bar{z}_{s} \wedge \frac{\sqrt{-1}}{2 \pi} \sum_{k, l=1}^{n} a_{k \bar{l}} d z_{k} \wedge d \bar{z}_{l} \wedge \omega^{n-2} \\
& =\left(\frac{\sqrt{-1}}{2 \pi}\right)^{n}(-1)^{\frac{1}{2}(n-1)(n-2)}(-1)^{n-1} A_{r \bar{s}} d z_{1} \wedge \cdots \wedge d \bar{z}_{n} .
\end{aligned}
$$

We also have the following algebraic fact:

$$
\begin{aligned}
& \frac{\sqrt{-1}}{2 \pi} d z_{r} \wedge d \bar{z}_{s} \wedge \frac{\sqrt{-1}}{2 \pi} \sum_{k, l=1}^{n} a_{k \bar{l}} d z_{k} \wedge d \bar{z}_{l} \wedge \omega^{n-2} \\
& =\frac{1}{n(n-1)}\left(\sum_{\alpha, \beta=1}^{n}\left(g^{\alpha \bar{\beta}} a_{\alpha \bar{\beta}}\right) g^{r \bar{s}}-\sum_{\alpha, \beta=1}^{n} g^{\alpha \bar{s}} g^{r \bar{\beta}} a_{\alpha \bar{\beta}}\right) \omega^{n} .
\end{aligned}
$$


By (2.5), we have

$$
\omega^{n}=\left(\frac{\sqrt{-1}}{2 \pi}\right)^{n} n !(-1)^{\frac{1}{2} n(n-1)} \frac{1}{\left(1+|z|^{2}\right)^{n+1}} d z_{1} \wedge \cdots \wedge d \bar{z}_{n} .
$$

Comparing (2.17), (2.18) and (2.19), we have

$$
A_{r \bar{s}}=\frac{(n-2) !}{\left(1+|z|^{2}\right)^{n+1}}\left(\sum_{\alpha, \beta=1}^{n}\left(g^{\alpha \bar{\beta}} a_{\alpha \bar{\beta}}\right) g^{r \bar{s}}-\sum_{\alpha, \beta=1}^{n} g^{\alpha \bar{s}} g^{r \bar{\beta}} a_{\alpha \bar{\beta}}\right),
$$

for $r, s=1, \cdots, n$. By $(2.20)$, we have

$$
\begin{aligned}
& \sum_{i, j=1}^{n} A_{i \bar{j}} b_{i} \bar{b}_{j}=\frac{(n-2) !}{\left(1+|z|^{2}\right)^{n+1}} \\
& \cdot\left(\sum_{\alpha, \beta=1}^{n} g^{\alpha \bar{\beta}} a_{\alpha \bar{\beta}} \sum_{i, j=1}^{n} g^{i \bar{j}} b_{i} \bar{b}_{j}-\sum_{i, j, \alpha, \beta=1}^{n} g^{\alpha \bar{j}} g^{i \bar{\beta}} a_{\alpha \bar{\beta}} b_{i} \bar{b}_{j}\right) .
\end{aligned}
$$

We need the following

Lemma 2.3. Using the same notations as above, we have

$$
\begin{aligned}
& \sum_{\alpha, \beta=1}^{n} g^{\alpha \bar{\beta}} a_{\alpha \bar{\beta}}=\left|Z_{0}\right|^{2}\left(1+|z|^{2}\right) \sum_{i=0}^{n} \tilde{a}_{i \bar{i}}, \\
& \sum_{i, j=1}^{n} g^{i \bar{j}} b_{i} \bar{b}_{j}=\left(1+|z|^{2}\right) \frac{|\nabla F|^{2}}{\left|F_{1}\right|^{2}}, \\
& \sum_{i, j, \alpha, \beta=1}^{n} g^{\alpha \bar{j}} g^{i \bar{\beta}} a_{\alpha \bar{\beta}} b_{i} \bar{b}_{j}=\left|Z_{0}\right|^{2}\left(1+|z|^{2}\right)^{2} \frac{\sum_{\alpha, \beta=0}^{n} \tilde{a}_{\alpha \bar{\beta}} \bar{F}_{\alpha} F_{\beta}}{\left|F_{1}\right|^{2}},
\end{aligned}
$$

where $\tilde{a}_{i \bar{j}}$ is defined in (2.12).

Proof. Comparing (2.12) and (2.16), we have

$$
\begin{cases}a_{k \bar{l}}=\tilde{a}_{k \bar{l}} \cdot\left|Z_{0}\right|^{2}, & k, l \neq 0 \\ \sum_{i=1}^{n} z_{i} a_{i \bar{l}}=-\tilde{a}_{0 \bar{l}} \cdot\left|Z_{0}\right|^{2}, & l \neq 0 \\ \sum_{j=1}^{n} \bar{z}_{j} a_{k \bar{j}}=-\tilde{a}_{k \overline{0}} \cdot\left|Z_{0}\right|^{2}, & k \neq 0 \\ \sum_{i, j=1}^{n} z_{i} \bar{z}_{j} a_{i \bar{j}}=\tilde{a}_{0 \overline{0}} \cdot\left|Z_{0}\right|^{2} . & \end{cases}
$$

Since $g^{\alpha \bar{\beta}}=\left(1+|z|^{2}\right)\left(\delta_{\alpha \beta}+z_{\alpha} \bar{z}_{\beta}\right)$, by (2.25), we have

$$
\sum_{\alpha, \beta=1}^{n} g^{\alpha \bar{\beta}} a_{\alpha \bar{\beta}}=\left(1+|z|^{2}\right) \sum_{\alpha, \beta=1}^{n}\left(\delta_{\alpha \beta}+z_{\alpha} \bar{z}_{\beta}\right) a_{\alpha \bar{\beta}}=|Z|^{2} \sum_{\alpha=0}^{n} \tilde{a}_{\alpha \bar{\alpha}}
$$


This proves (2.22). By (2.10), we have

$$
\sum_{i=1}^{n} z_{i} b_{i}=-\frac{F_{0}}{F_{1}}
$$

on $M$. Thus (2.23) and (2.24) follow from a straightforward computation using the above equation.

Continuation of the Proof of Lemma 2.2. By Lemma 2.3, we have

$$
\begin{aligned}
& \sum_{\alpha, \beta=1}^{n} g^{\alpha \bar{\beta}} a_{\alpha \bar{\beta}} \sum_{i, j=1}^{n} g^{i \bar{j}} b_{i} \bar{b}_{j}-\sum_{i, j, \alpha, \beta=1}^{n} g^{\alpha \bar{j}} g^{i \bar{\beta}} a_{\alpha \bar{\beta}} b_{i} \bar{b}_{j} \\
& =\left|Z_{0}\right|^{2}\left(1+|z|^{2}\right)^{2} \frac{|\nabla F|^{2}}{\left|F_{1}\right|^{2}}\left(\sum_{i=0}^{n} \tilde{a}_{i \bar{i}}-\sum_{i, j=0}^{n} \frac{\tilde{a}_{i \bar{j}} F_{j} \bar{F}_{i}}{|\nabla F|^{2}}\right) .
\end{aligned}
$$

By (2.11),

$$
\begin{aligned}
\omega^{n-1} & =\left(\frac{\sqrt{-1}}{2 \pi}\right)^{n-1}(-1)^{\frac{1}{2}(n-1)(n-2)} \frac{(n-1) !}{\left(1+|z|^{2}\right)^{n}} \frac{|\nabla F|^{2}}{\left|F_{1}\right|^{2}} \\
& \cdot d z_{2} \wedge \cdots \wedge d z_{n} \wedge d \bar{z}_{2} \wedge \cdots d \bar{z}_{n} .
\end{aligned}
$$

(2.13) follows from (2.15), (2.21), (2.26) and (2.27).

Lemma 2.4. Let $\xi$ be the function defined in (2.3) and let $\theta$ be defined in (2.2). Then we have

$$
\begin{aligned}
& \frac{\sqrt{-1}}{2 \pi} \partial \xi \wedge \bar{\partial} \theta \wedge \omega^{n-2} \\
= & \frac{1}{n-1}\left(-\sum_{k=0}^{n}\left(\frac{X F}{|\nabla F|^{2}}\right)_{k} \overline{F_{k}}+\frac{\sum_{k=0}^{n} \lambda_{k}\left|F_{k}\right|^{2}}{|\nabla F|^{2}}-(d-1) \theta\right) \omega^{n-1} .
\end{aligned}
$$

Furthermore, we have

$$
\begin{aligned}
& \frac{\sqrt{-1}}{2 \pi} \int_{M} \partial \xi \wedge \bar{\partial} \theta \wedge \omega^{n-2} \\
= & \frac{1}{n-1} \int_{M} \sum_{k=0}^{n}\left(\frac{X F}{|\nabla F|^{2}}\right)_{k} \overline{F_{k}} \omega^{n-1}+\frac{n-d+1}{n-1} \int_{M} \theta \omega^{n-1} .
\end{aligned}
$$


Proof. Let $\eta=\frac{\sqrt{-1}}{2 \pi} \partial \xi \wedge \bar{\partial} \theta$ and let

$$
\pi^{*} \eta=\frac{\sqrt{-1}}{2 \pi} \sum_{i, j=0}^{n} \tilde{a}_{i \bar{j}} d Z_{i} \wedge d \bar{Z}_{j}
$$

Then we have

$$
\tilde{a}_{i \bar{j}}=\frac{\partial \xi}{\partial Z_{i}} \cdot \frac{\partial \theta}{\partial \bar{Z}_{j}}
$$

A straightforward computation gives

$$
\sum_{i=0}^{n} \tilde{a}_{i \bar{i}}=\frac{-\sum_{k=0}^{n}(X F)_{k} \bar{F}_{k}+\sum_{k=0}^{n} \lambda_{k}\left|F_{k}\right|^{2}}{|Z|^{2}|\nabla F|^{2}}-(d-1) \frac{\theta}{|Z|^{2}},
$$

and

$$
\frac{\sum_{i, j=0}^{n} \tilde{a}_{i j} F_{j} \bar{F}_{i}}{|\nabla F|^{2}}=-\frac{X F \cdot \sum_{i, k=0}^{n} F_{i k} \bar{F}_{i} \bar{F}_{k}}{|Z|^{2}|\nabla F|^{4}}
$$

on $M$. Thus by Lemma 2.2, we got (2.28). Using Lemma 2.2 again for $\eta=\frac{\sqrt{-1}}{2 \pi} \partial \bar{\partial} \theta$, we have

$$
\frac{\sqrt{-1}}{2 \pi} \partial \bar{\partial} \theta \wedge \omega^{n-2}=\frac{1}{n-1}\left(-n \theta+\frac{\sum_{k=0}^{n} \lambda_{k}\left|F_{k}\right|^{2}}{|\nabla F|^{2}}\right) \omega^{n-1} .
$$

(2.29) follows from (2.28), (2.30) and the Stokes Theorem.

Although not needed in this paper, we give a simple proof of the following formula for the Futaki invariant in [5] as an application of Lemma 2.1, Lemma 2.2 and Lemma 2.4.

Corollary 2.1. Let $M$ be a smooth hypersurface in $C P^{n}$ defined by the homogeneous polynomial $F=0$ of degree $d$. Let $X$ be a vector in $C P^{n}$ satisfying

$$
X F=\kappa F .
$$

The Futaki invariant is defined as

$$
\mathcal{F}(X)=-\int_{M} X(\xi) \omega^{n-1} .
$$

Then

$$
\mathcal{F}(X)=-\frac{(n+1)(d-1)}{n} \kappa .
$$


Proof. We have

$$
i(X) \omega=-\frac{\sqrt{-1}}{2 \pi} \bar{\partial} \theta
$$

Since $X$ leave $M$ invariant, we have

$$
0=\int_{M} i(X)\left(\partial \xi \wedge \omega^{n-1}\right)=\int_{M} X \xi \omega^{n-1}+(n-1) \int_{M} \frac{\sqrt{-1}}{2 \pi} \partial \xi \wedge \bar{\partial} \theta \wedge \omega^{n-2} .
$$

By the above equation and (2.31), from Lemma 2.4, we have

$$
\mathcal{F}(X)=-\kappa \int_{M} \omega^{n-1}+(n-d+1) \int_{M} \theta \omega^{n-1}
$$

By [5, Theorem 5.1], we have

$$
\int_{M} \theta \omega^{n-1}=\frac{\kappa}{n}
$$

(2.32) follows from the above two equations.

Finally, we have the following

Theorem 2.5. The $K$ energy $\mathcal{M}(t)$ can be represented as

$$
\begin{aligned}
& \mathcal{M}(t)=\frac{2}{d} \int_{1}^{t}\left(\int _ { M _ { \tau } } \frac { 1 } { \tau } \left(-\sum_{k=0}^{n}\left(\frac{X F_{\tau}}{\left|\nabla F_{\tau}\right|^{2}}\right)_{k} \overline{\left(F_{\tau}\right)_{k}} \omega^{n-1}\right.\right. \\
& \left.\left.+(n-d+1) \int_{M_{\tau}} \theta \omega^{n-1}\right)\right) d \tau
\end{aligned}
$$

where

$$
F_{\tau}\left(Z_{0}, \cdots, Z_{n}\right)=F\left(\tau^{-\lambda_{0}} Z_{0}, \cdots, \tau^{-\lambda_{n}} Z_{n}\right),
$$

and $M_{\tau}$ is the zero set of $F_{\tau}=0$. In particular, we have

$$
\begin{aligned}
& t \frac{d}{d t} \mathcal{M}(t)=\frac{2}{d}\left(-\int_{M_{t}} \sum_{k=0}^{n}\left(\frac{X F_{t}}{\left|\nabla F_{t}\right|^{2}}\right)_{k} \overline{\left(F_{t}\right)_{k}} \omega^{n-1}\right. \\
& \left.+(n-d+1) \int_{M_{t}} \theta \omega^{n-1}\right) .
\end{aligned}
$$

Proof. The theorem follows from Proposition 2.2, Lemma 2.1 and Lemma 2.4. 


\section{The limit of the derivative of the $K$ energy.}

In this section, we compute the limit $\lim _{t \rightarrow 0} t \mathcal{M}^{\prime}(t)$ using Theorem 2.5. First, we need some combinatoric preparations.

Let $\left(\delta_{i}, \sigma_{i}\right), i=0, \cdots, p$ be a sequence of pair of nonnegative rational numbers. $\delta_{0}=0$. We assume that the sequence is "generic" in the sense that

1. All $\delta_{i},(i=0, \cdots, p)$ are distinct numbers(that implies $\delta_{i}>0, i=$ $1, \cdots, p)$;

2. None of the three lines defined by $\psi_{i}(x)=\delta_{i}+\sigma_{i} x,(i=0, \cdots, p)$ intersect at the same point.

Define $\left(i_{k}, r_{k}\right),(k=0, \cdots, m)$ inductively as follows: let $i_{0}=0, r_{0}=0$. If $\left(i_{k}, r_{k}\right)$ has been defined, then

1. If for any $r>r_{k}$

$$
\delta_{i_{k}}+\sigma_{i_{k}} r<\delta_{i}+\sigma_{i} r \quad\left(i \neq i_{k}\right)
$$

then let $m=k$ and stop;

2. If not, then define $i_{k+1}$ and $r_{k+1}>r_{k}$ such that

$$
\delta_{i_{k}}+\sigma_{i_{k}} r_{k+1}=\delta_{i_{k+1}}+\sigma_{i_{k+1}} r_{k+1} \leq \delta_{i}+\sigma_{i} r_{k+1},
$$

where $i=1, \cdots, p$. Since $\left(\delta_{i}, \sigma_{i}\right), i=0, \cdots, p$ are "generic", the choice of $\left(i_{k}, r_{k}\right)$ is unique for $(k=0, \cdots, m)$.

We have the following obvious

Lemma 3.1. $\left(i_{k}, r_{k}\right),(k=0,1, \cdots)$ is a finite sequence. In particular, the sequence stops at $\left(i_{m}, r_{m}\right)$.

Proof. By the construction of $i_{k}$ 's, we have

$$
\sigma_{i_{0}}>\sigma_{i_{1}}>\cdots>\sigma_{i_{k}}>\cdots .
$$

Thus all $i_{k}$ 's must be distinct. But $0 \leq i_{k} \leq p$. So the length of the sequence is at most $p+1$.

Let

$$
\psi(x)=\operatorname{Min}_{i \geq 0}\left(\delta_{i}+\sigma_{i} x\right)
$$

The function $\psi(x)$ is a piecewise linear function, whose derivative exists almost everywhere. $r_{k},(k=1, \cdots, m)$ are the non-smooth points of $\psi(x)$. 
Lemma 3.2. Assuming that $\sigma_{i_{m}}=0$, we have

$$
\sum_{k=0}^{m-1}\left(-\delta_{i_{k}}+\delta_{i_{k+1}}\right)\left(\sigma_{i_{k}}+\sigma_{i_{k+1}}-1\right)=\int_{0}^{\infty} \psi^{\prime}(x)\left(\psi^{\prime}(x)-1\right) d x .
$$

Proof. First, let's remark that for $x$ large enough, $\psi \equiv \delta_{i_{m}}$ is a constant. Thus the integral in the lemma is convergent.

By definition of $r_{k}(k=0, \cdots, m)$ in $(3.1)$, we have

$$
-\delta_{i_{k}}+\delta_{i_{k+1}}=\left(\sigma_{i_{k}}-\sigma_{i_{k+1}}\right) r_{k+1}
$$

for $k=0, \cdots, m-1$. Thus we have

$$
\sum_{k=0}^{m-1}\left(-\delta_{i_{k}}+\delta_{i_{k+1}}\right)\left(\sigma_{i_{k}}+\sigma_{i_{k+1}}-1\right)=\sum_{k=0}^{m-1} r_{k+1}\left(\sigma_{i_{k}}^{2}-\sigma_{i_{k+1}}^{2}\right)+\left(\delta_{i_{0}}-\delta_{i_{m}}\right) .
$$

The second term of the above equation is equal to

$$
-\int_{0}^{\infty} \psi^{\prime}(x) d x
$$

For the first term, using the summation by parts, we have

$$
\sum_{k=0}^{m-1} r_{k+1}\left(\sigma_{i_{k}}^{2}-\sigma_{i_{k+1}}^{2}\right)=r_{1}\left(\sigma_{i_{0}}\right)^{2}+\sum_{k=1}^{m-1} \sigma_{i_{k}}^{2}\left(r_{k+1}-r_{k}\right)=\int_{0}^{\infty} \psi^{\prime}(x)^{2} d x .
$$

Combining the above two equations, we get (3.3).

Consider the smooth hypersurface $M \subset C P^{n}$ defined by the polynomial $F=0$ of degree $d$. Let $X=\sum_{i=0}^{n} \lambda_{i} Z_{i} \frac{\partial}{\partial Z_{i}}$ be the vector field for integers $\left(\lambda_{0}, \cdots, \lambda_{n}\right)$ such that $\sum \lambda_{i}=0$. Let $M_{t}$ be defined by the equation

$$
F_{t}\left(Z_{0}, \cdots, Z_{n}\right)=F\left(t^{-\lambda_{0}} Z_{0}, \cdots, t^{-\lambda_{n}} Z_{n}\right) .
$$

We write $F_{t}$ as

$$
F_{t}=t^{\delta} \sum_{i=0}^{p} a_{i} t^{\delta_{i}} Z_{0}^{\alpha_{0}^{i}} \cdots Z_{n}^{\alpha_{n}^{i}}
$$

where $\delta_{0}=0$, and $\delta_{i} \geq 0, i=1, \cdots, p$. By (3.4), we have

$$
X\left(Z_{0}^{\alpha_{0}^{i}} \cdots Z_{n}^{\alpha_{n}^{i}}\right)=-\left(\delta_{i}+\delta\right) Z_{0}^{\alpha_{0}^{i}} \cdots Z_{n}^{\alpha_{n}^{i}}
$$

for $i=0, \cdots, p$.

In what follows we assume that the choice of $\left(\lambda_{0}, \cdots, \lambda_{n}\right)$ is "generic" in the following sense: 
1. All $\delta_{i}$ 's are distinct;

2. None of the three lines defined by $\delta_{i}+\alpha_{k}^{i} x$ for $i=0, \cdots, p$ intersect at the same points, where $k=0, \cdots, n$.

Without losing generality, we may assume that $a_{0}=1$, and $0=\delta_{0}<\delta_{1}<$ $\delta_{2}<\cdots<\delta_{p}$. We also assume that $a_{0}, \cdots, a_{p}$ are all non-zero. Furthermore, since $M$ is smooth, we see that for each $0 \leq k \leq n$, there is an $0 \leq i \leq p$ such that $\alpha_{k}^{i}=0$.

Let $U_{i}=\left\{\left[Z_{0}, \cdots, Z_{n}\right] \in C P^{n}|| Z_{i}\left|>\frac{1}{2}\right| Z_{j} \mid, j=0, \cdots, n\right\}$. Then $\cup U_{i}=$ $C P^{n}$. Let $P_{i}=\left\{Z_{i}=0\right\}$ and $P_{i j}=P_{i} \cap P_{j}$ for $i \neq j$ and $i, j=0, \cdots, n$. Let $\sigma>0$ be chosen so that $\sigma<\frac{1}{d} \operatorname{Min}_{i \geq 1}\left(\delta_{i}\right)$ (Note that $\underset{i \geq 1}{\operatorname{Min}}\left(\delta_{i}\right)>0$ ) and define

$$
V_{i j}^{t}=\left\{\left.z \in C P^{n}\left|d\left(z, P_{i j}\right)<\right| t\right|^{\sigma}\right\}, i \neq j, i, j=0, \cdots, n,
$$

where $d(\cdot, \cdot)$ is the distance induced by the Fubini-Study metric on $C P^{n}$.

By (3.5), we see that $t^{-\delta} F_{t} \rightarrow Z_{0}^{\alpha_{0}^{0}} \cdots Z_{n}^{\alpha_{n}^{0}}$ as $t \rightarrow 0$. Intuitively, $M_{t}$ goes to the hyperplanes defined by $Z_{0}^{\alpha_{0}^{0}} \cdots Z_{n}^{\alpha_{n}^{0}}=0$. This turns out to be essentially true by the following Lemma:

Lemma 3.3. There is a $\sigma_{1}>\sigma$ such that for any $0 \leq k \leq n$ and

$$
\left[Z_{0}, \cdots, Z_{n}\right] \in\left(M_{t}-\cup_{i, j=0}^{n} V_{i j}^{t}\right) \cap U_{k},
$$

one can find a unique $l \neq k$ such that

$$
\left|\frac{Z_{l}}{Z_{k}}\right|<|t|^{\sigma_{1}}
$$

for $t$ small enough, where $\left[Z_{0}, \cdots, Z_{n}\right] \in M_{t}$.

Proof. By (3.5) we have

$$
\left|Z_{0}^{\alpha_{0}^{0}} \cdots Z_{n}^{\alpha_{n}^{0}}\right| \leq 2^{d} \sum_{i=1}^{p}\left|a_{i}\right||t|^{\operatorname{Min}\left(\delta_{i}\right)}\left|Z_{k}\right|^{d} .
$$

Thus if for any $l \neq k$,

$$
\left|\frac{Z_{l}}{Z_{k}}\right| \geq|t|^{\sigma_{1}}
$$

we could have

$$
\left|Z_{0}^{\alpha_{0}^{0}} \cdots Z_{n}^{\alpha_{n}^{0}}\right| \geq|t|^{\sigma_{1} d}\left|Z_{k}\right|^{d} .
$$


This is a contradiction since we choose $\sigma_{1}$ such that

$$
\sigma<\sigma_{1}<\frac{1}{d} \operatorname{Min}_{i \geq 1}\left(\delta_{i}\right)
$$

We are now going to prove that for $t$ small enough, the connected components of $M_{t} \backslash \cup V_{i j}^{t}$ are graphs of functions over $\tilde{P}_{i}$, where

$$
\tilde{P}_{i}=P_{i}-\cup_{j \neq i} V_{i j}^{t}
$$

To see this, we let

$$
Q_{i}=\left\{\left[Z_{0}, \cdots, Z_{i}, \cdots, Z_{n}\right] \mid\left[Z_{0}, \cdots, Z_{i-1}, \underset{i}{0}, Z_{i+1}, \cdots, Z_{n}\right] \in \tilde{P}_{i}\right\},
$$

for $i=0, \cdots, n$. By (1.4) and (1.5), we have

$$
\varphi\left(x_{0}, \cdots, x_{n}\right)=\operatorname{Min}_{0 \leq i \leq p}\left(\delta+\delta_{i}+\alpha_{0}^{i} x_{0}+\cdots+\alpha_{n}^{i} x_{n}\right)
$$

and

$$
\varphi_{k}(x)=\operatorname{Min}_{0 \leq i \leq p}\left(\delta+\delta_{i}+\alpha_{k}^{i} x\right)
$$

for $k=0, \cdots, n$.

Remark 3.1. $\varphi$ and $\varphi_{k}(k=0, \cdots, n)$ are defined even if $\lambda_{0}, \cdots, \lambda_{n}$ are not choosing "generically". In the special case when

$$
X F=\kappa F,
$$

we have

$$
\varphi_{i}(x)=-\kappa+\left(\operatorname{Min}_{0 \leq j \leq p} \alpha_{i}^{j}\right) x
$$

for $0 \leq i \leq n$ and $x \geq 0$. If $M$ is a normal variety, we have

$$
\operatorname{Min}_{0 \leq j \leq p} \alpha_{i}^{j}=0 \text { or } 1
$$

In particular, in this case

$$
\varphi_{i}^{\prime}(x)\left(\varphi_{i}^{\prime}(x)-1\right)=0
$$

for $0 \leq i \leq n$. Using this and Theorem 1.1, we recover the main result in [2] for hypersurfaces. 
Proposition 3.1. Using the notations as above, we have

$$
\begin{aligned}
& \int_{M_{t} \cap Q_{i}} \sum_{A=0}^{n}\left(\frac{X F_{t}}{\left|\nabla F_{t}\right|^{2}}\right)_{A} \overline{\left(F_{t}\right)_{A}} \omega^{n-1} \\
& \rightarrow-\delta \alpha_{i}^{0}-\int_{0}^{\infty} \varphi_{i}^{\prime}(x)\left(\varphi_{i}^{\prime}(x)-1\right) d x,
\end{aligned}
$$

for $i=0, \cdots, n$ as $t \rightarrow 0$.

Proof. For the sake of simplicity, we omit unimportant constants in an inequality. Thus in the proof of this proposition, $A \leq B$ means there is a constant $C$ independent of $t$ such that $A \leq C B$.

We just need to prove the theorem for the case $i=1$. If $\alpha_{1}^{0}=0$, then the proposition is automatically true since $\varphi_{1}^{\prime} \equiv 0$. Thus we assume that $\alpha_{1}^{0} \geq 1$. We work on $M_{t} \cap Q_{1} \cap U_{0}$, without losing generality.

We assume that $\left(z_{1}, \cdots, z_{n}\right)=\left(\frac{Z_{1}}{Z_{0}}, \cdots, \frac{Z_{n}}{Z_{0}}\right)$ on $U_{0}$. Then $F_{t}=0$ can be written as

$$
f=\sum_{i=0}^{p} a_{i} t^{\delta_{i}} z_{1}^{\alpha_{1}^{i}} \cdots z_{n}^{\alpha_{n}^{i}}=0
$$

with $a_{0}=1$ and $\delta_{0}=0($ see $(3.5))$. The sequence $\left(\delta_{i}, \alpha_{1}^{i}\right),(i=0, \cdots, p)$ is assumed to be a "generic" sequence mentioned at the beginning of this section.

For $\left(z_{1}, \cdots, z_{n}\right) \in \tilde{P}_{1} \cap U_{0}$, we have

$$
\left|z_{i}\right| \geq|t|^{\sigma}
$$

for $i=2, \cdots, n$. Let $\xi_{i}^{k}\left(i=1, \cdots, \alpha_{1}^{i_{k}}-\alpha_{1}^{i_{k+1}}, k=0, \cdots, m\right)$ be the $\left(\alpha_{1}^{i_{k}}-\right.$ $\alpha_{1}^{i_{k+1}}$ )-th roots of

$$
-\frac{a_{i_{k+1}}}{a_{i_{k}}} t^{\delta_{i_{k+1}}-\delta_{i_{k}}} z_{2}^{\alpha_{2}^{i_{k+1}}-\alpha_{2}^{i_{k}}} \cdots z_{n}^{\alpha_{n}^{i_{k+1}}-\alpha_{n}^{i_{k}}} .
$$

In the following lemma, we give the solutions of $z_{1}=z_{1}\left(z_{2}, \cdots, z_{n}\right)$ of the equation $f=0$. Of course, they are multiple solutions.

Lemma 3.4. For $\sigma>0$ small enough, there is a constant $\varepsilon_{0}>0$ such that the solutions of $z_{1}$ of $f=0$ satisfies

$$
\left|z_{1}-\xi_{i}^{k}\right| \leq\left|\xi_{i}^{k}\right| \cdot|t|^{\varepsilon_{0}}
$$

for $\left(i=1, \cdots, \alpha_{1}^{i_{k}}-\alpha_{1}^{i_{k+1}}, k=0, \cdots, m-1\right)$. Furthermore, the balls $B_{i}^{k}=$ $\left\{z \in \mathbb{C}|| z-\left.\xi_{i}^{k}|\leq| \xi_{i}^{k}|| t\right|^{\varepsilon_{0}}\right\}$ for $\left(i=1, \cdots, \alpha_{1}^{i_{k}}-\alpha_{1}^{i_{k+1}}, k=0, \cdots, m-1\right) d o$ not intersect each other. 
Proof. In the proof, the scripts $i, k$ are always running in $\left(i=1, \cdots, \alpha_{1}^{i_{k}}-\right.$ $\left.\alpha_{1}^{i_{k+1}}, k=0, \cdots, m-1\right)$, unless otherwise stated. We choose $\varepsilon_{1}>0$ such that

$$
\varepsilon_{1}<\operatorname{Min}_{0 \leq k \leq m} \operatorname{Min}_{i \neq i_{k}, i_{k+1}}\left(\delta+\delta_{i}+\alpha_{1}^{i} r_{k+1}-\varphi_{1}\left(r_{k+1}\right)\right) .
$$

Define $f_{k}$ and $g_{k}$ as follows

$$
f_{k}=a_{i_{k}} t^{\delta_{i_{k}}} z_{1}^{\alpha_{1}^{i_{k}}} \cdots z_{n}^{\alpha_{n}^{i_{k}}}+a_{i_{k+1}} t^{\delta_{i_{k+1}}} z_{1}^{\alpha_{1}^{i_{k+1}}} \cdots z_{n}^{\alpha_{n}^{i_{k+1}}}
$$

and

$$
g_{k}=f-f_{k} .
$$

By the definition of $\xi_{i}^{k}$, we have

$$
|t|^{r_{k+1}+C \sigma} \leq\left|\xi_{i}^{k}\right| \leq|t|^{r_{k+1}-C \sigma}
$$

for some constant $C$ independent of $t$. We also have

$$
|t|^{\delta}\left|g_{k}\right| \leq|t|^{\varphi_{1}\left(r_{k+1}\right)+\varepsilon_{1}-d \sigma}
$$

on $B_{i}^{k}$ and

$$
|t|^{\delta}\left|f_{k}\right| \geq|t|^{\varphi_{1}\left(r_{k+1}\right)+\varepsilon_{0}+d \sigma}
$$

on $\partial B_{i}^{k}$. We choose $\sigma$ small enough such that $\varepsilon_{1}-d \sigma>\frac{3}{4} \varepsilon_{1}$ and $\varepsilon_{0}$ small enough such that $\varepsilon_{0} \leq \frac{1}{4} \varepsilon_{1}$ Thus we have

$$
\left|f_{k}\right|>\left|g_{k}\right|
$$

on $\partial B_{i}^{k}$. By the Rouché Theorem, $f_{k}$ and $f=f_{k}+g_{k}$ have the same number of solutions in $B_{i}^{k}$. Since $f_{k}$ has only one solution in $B_{i}^{k}$, we prove the first claim of the lemma. Next, if $t$ is small enough, we have a constant $C$ such that

$$
\left|\xi_{i}^{k}-\xi_{i_{1}}^{k_{1}}\right| \geq C \operatorname{Max}\left(\left|\xi_{i}^{k}\right|,\left|\xi_{i_{1}}^{k_{1}}\right|\right)
$$

Thus if $t$ is small enough, $B_{i}^{k}$ 's do not intersect each other.

Continuation of the proof of Proposition 3.1. For simplicity, let $F=$ $F_{t}$. For fixed $i, k$, attaching the $B_{i}^{k}$ in the above lemma for each $p \in \tilde{P}_{1} \cap U_{0}$, 
we get a bundle $\tilde{B}_{i}^{k}$. On each bundle $\tilde{B}_{i}^{k}$, since $\left|z_{i}\right|>|t|^{\sigma}$, we have

$$
\begin{gathered}
\sum_{A=0}^{n}\left(\frac{X F}{|\nabla F|^{2}}\right)_{A}\left(\overline{F_{A}}\right)=\frac{(X F)_{1}}{F_{1}}-\frac{(X F) F_{11}}{F_{1}^{2}}+o(1) \\
=\frac{-\left(\delta+\delta_{i_{k}}\right) \alpha_{1}^{i_{k}}+\left(\delta+\delta_{i_{k+1}}\right) \alpha_{1}^{i_{k+1}}}{\alpha_{1}^{i_{k}}-\alpha_{1}^{i_{k+1}}} \\
-\frac{\left(-\delta_{i_{k}}+\delta_{i_{k+1}}\right)\left(\alpha_{1}^{i_{k}}\left(\alpha_{1}^{i_{k}}-1\right)-\alpha_{1}^{i_{k+1}}\left(\alpha_{1}^{i_{k+1}}-1\right)\right)}{\left(\alpha_{1}^{i_{k}}-\alpha_{1}^{i_{k+1}}\right)^{2}}+o(1) \\
=-\delta+\frac{-\delta_{i_{k}} \alpha_{1}^{i_{k}}+\delta_{i_{k+1}} \alpha_{1}^{i_{k+1}}+\left(\delta_{i_{k}}-\delta_{i_{k+1}}\right)\left(\alpha_{1}^{i_{k}}+\alpha_{1}^{i_{k+1}}-1\right)}{\alpha_{1}^{i_{k}}-\alpha_{1}^{i_{k+1}}}+o(1)
\end{gathered}
$$

as $t \rightarrow 0$ for $k=0, \cdots, m-1$, where $o(1)$ is a quantity that goes to zero as $t \rightarrow 0$. By the same argument, the above equation is also true for $p \in \tilde{P}_{1} \cap U_{l}$ for $l \neq 0$. Thus the equation is true for $p \in \tilde{P}_{1}$. On the other hand, by (3.11), we have

$$
\operatorname{det} \pi=o(1)
$$

as $t \rightarrow 0$, where $\pi: Q_{1} \rightarrow \tilde{P}_{1}$ is the projection. This is because $\frac{\partial z_{1}}{\partial z_{k}}=$ $-F_{k} / F_{1} \rightarrow 0$ for $x \in \tilde{P}_{1}$. Thus by (3.12) and (3.13), we have

$$
\begin{gathered}
\int_{M_{t} \cap Q_{1}} \sum_{A=0}^{n}\left(\frac{X F_{t}}{\left|\nabla F_{t}\right|^{2}}\right)_{A}\left(\overline{\left.F_{t}\right)_{A}} \omega^{n-1}\right. \\
=\left(-\delta \alpha_{1}^{0}+\sum_{k=0}^{m-1}\left(\delta_{i_{k}}-\delta_{i_{k+1}}\right)\left(\alpha_{1}^{i_{k}}+\alpha_{1}^{i_{k+1}}-1\right)\right) \operatorname{vol}\left(C P^{n-1}\right)+o(1)
\end{gathered}
$$

as $t \rightarrow 0$. The proposition follows from Lemma 3.2 and the fact $\operatorname{vol}\left(C P^{n-1}\right)=1$.

Lemma 3.5. Let $p$ be a fixed point in $M_{t}$ and let $d(x, p)$ be the distance from $x \in C P^{n}$ to $p$ defined by the Fubini-Study metric. Let $B_{p}(\varepsilon)=\{x \in$ $\left.C P^{n} \mid d(x, p)<\varepsilon\right\}$. Then there are constants $C, \sigma$ independent of $p$ and $t$ such that

$$
\int_{M_{t} \cap B_{p}(\varepsilon)} \omega^{n-1} \leq C \varepsilon^{2 n-2} \log \varepsilon^{-1}
$$

for $t$ small enough, where $\varepsilon=|t|^{\sigma}$. 
Proof. Consider the cut-off function $\rho: \mathbb{R} \rightarrow \mathbb{R}$ such that $\rho \geq 0$ is smooth, $\rho \equiv 1$ on $[0,1]$ and $\rho \equiv 0$ on $(-\infty,-1] \cup[2,+\infty)$. Then we have

$$
\int_{M_{t} \cap B_{p}(\varepsilon)} \omega^{n-1} \leq \int_{M_{t}} \rho\left(\frac{d(x, p)}{\varepsilon}\right) \omega^{n-1} .
$$

Let $F_{t}$ be the defining function of $M_{t}$. Then in the sense of distribution, we have

$$
\frac{\sqrt{-1}}{2 \pi} \partial \bar{\partial} \log \frac{\left|F_{t}\right|^{2}}{\left(\sum_{i=0}^{n}\left|Z_{i}\right|^{2}\right)^{d}}=\left[M_{t}\right]-d \omega
$$

Thus we have

$$
\begin{aligned}
\int_{M_{t}} \rho\left(\frac{d(x, p)}{\varepsilon}\right) \omega^{n-1}= & d \int_{C P^{n}} \rho\left(\frac{d(x, p)}{\varepsilon}\right) \omega^{n} \\
& +\int_{C P^{n}} \rho \frac{\sqrt{-1}}{2 \pi} \partial \bar{\partial} \log \frac{\left|F_{t}\right|^{2}}{\left(\sum_{i=0}^{n}\left|Z_{i}\right|^{2}\right)^{d}} \omega^{n-1} .
\end{aligned}
$$

We have an easy estimate for the first term of the right hand side of (3.15):

$$
\int_{C P^{n}} \rho\left(\frac{d(x, p)}{\varepsilon}\right) \omega^{n} \leq C \varepsilon^{2 n}
$$

For the second term, assume that $p \in U_{0}=\left\{\left[Z_{0}, \cdots, Z_{n}\right]|| Z_{0}\left|>\frac{1}{2}\right| Z_{j} \mid, j=\right.$ $1, \cdots, n\}$. Then by $(3.5)$

$$
F_{t}=t^{\delta} Z_{0}^{d} f_{t}
$$

where $f_{t} \rightarrow f_{0}=z_{1}^{\alpha_{1}^{0}} \cdots z_{n}^{\alpha_{n}^{0}} \not \equiv 0$. Thus using integration by parts, we have

$$
\begin{aligned}
& \int_{C P^{n}} \rho\left(\frac{d(x, p)}{\varepsilon}\right) \frac{\sqrt{-1}}{2 \pi} \partial \bar{\partial} \log \frac{\left|F_{t}\right|^{2}}{\left(\sum_{i=0}^{n}\left|Z_{i}\right|^{2}\right)^{d}} \omega^{n-1} \\
& \leq C \varepsilon^{2 n}+\frac{C}{\varepsilon^{2}}\left|\int_{|z| \leq 2 \varepsilon} \log \right| f_{t}\left|d V_{0}\right|
\end{aligned}
$$

where $d V_{0}=\left(\frac{\sqrt{-1}}{2 \pi}\right)^{n} d z_{1} \wedge d \bar{z}_{1} \wedge \cdots \wedge d \bar{z}_{n}$ is the Euclidean measure and $|z|^{2}=\left|z_{1}\right|^{2}+\cdots+\left|z_{n}\right|^{2}$. Rescaling the second term of the above integral, we have

$$
\frac{C}{\varepsilon^{2}}\left|\int_{|z| \leq 2 \varepsilon} \log \right| f_{t}\left|d V_{0}\right|=C \varepsilon^{2 n-2} \log \varepsilon^{-1}+C \varepsilon^{2 n-2}\left|\int_{|z| \leq 2} \log \right| \tilde{f}_{t}\left|d V_{0}\right|,
$$


where $\tilde{f}_{t}\left(z_{1}, \cdots, z_{n}\right)=f_{t}\left(\varepsilon z_{1}, \cdots, \varepsilon z_{n}\right) / \varepsilon^{\alpha_{1}^{0}+\cdots+\alpha_{n}^{0}}$. By (3.5), if $\sigma$ is small enough, we have $\tilde{f}_{t} \rightarrow z_{1}^{\alpha_{1}^{0}} \cdots z_{n}^{\alpha_{n}^{0}}$. By a theorem of Phong and Sturm [14], we have

$$
\int_{|z| \leq 2} \log \left|f_{t}\right|^{-1} d V_{0} \leq C
$$

for $t$ small enough. (3.14) follows from (3.15), (3.16), (3.17), and (3.19).

Lemma 3.6. There exists a constant $C>0$ such that for $t$ small

$$
\sum_{i \neq j} \int_{V_{i j}^{t} \cap M_{t}} \omega^{n-1} \leq C|t|^{2 \sigma} \log |t|^{-1} .
$$

Proof. Let $\varepsilon=|t|^{\sigma}$. Fixing $i, j$, there is a constant $C_{0}$ independent of $\varepsilon$ such that one can find points $p_{1}, \cdots, p_{m} \in P_{i j}$ for $m=\left[\frac{C_{0}}{\varepsilon^{2 n-4}}\right]$, satisfying

$$
\bigcup_{k=1}^{m} B_{p_{k}}(\varepsilon) \supset P_{i j}
$$

Thus by the above lemma, we have

$$
\int_{V_{i j}^{t} \cap M_{t}} \omega^{n-1} \leq \sum_{k=1}^{m} \int_{M_{t} \cap B_{p_{k}}\left(|t|^{\sigma}+\varepsilon\right)} \omega^{n-1} .
$$

By Lemma 3.5, we have

$$
\int_{V_{i j}^{t} \cap M_{t}} \omega^{n-1} \leq \frac{C}{\varepsilon^{2 n-4}} \varepsilon^{2 n-2} \log \varepsilon^{-1}=C \varepsilon^{2} \log \varepsilon^{-1} .
$$

The lemma follows since $\varepsilon=|t|^{\sigma}$.

Lemma 3.7. There exists a constant $C$ independent of $t$ such that for any measurable subset $E$ of $M_{t}$

$$
\left|\int_{E} \partial \xi \wedge \bar{\partial} \theta \wedge \omega^{n-2}\right| \leq C \sqrt{\log |t|^{-1}} \cdot \sqrt{\operatorname{meas}(E)},
$$

where the functions $\xi$ and $\theta$ are defined in (2.3) and (2.2), respectively.

Proof. Since $M_{t}$ is a submanifold, the Ricci curvature has an upper bound. Thus from (2.3), we have a constant $C$ such that

$$
-\frac{\sqrt{-1}}{2 \pi} \partial \bar{\partial} \xi \leq C \omega
$$


On the other hand, since $\left[t^{\lambda_{0}} Z_{0}, \cdots, t^{\lambda_{n}} Z_{n}\right] \in M_{t}$ iff $\left[Z_{0}, \cdots, Z_{n}\right] \in M$, we have

$$
\left|\nabla F_{t}\right|^{2}\left(t^{\lambda_{0}} Z_{0}, \cdots, t^{\lambda_{n}} Z_{n}\right)=\sum_{l=0}^{n}|t|^{-2 \lambda_{l}}\left|F_{l}\right|^{2}\left(Z_{0}, \cdots, Z_{n}\right) .
$$

Since $M$ is smooth, we have

$$
-C \log |t|^{-1} \leq|\xi| \leq C \log |t|^{-1}
$$

for some constant $C$. Using integration by parts, from (3.20), and the above estimate, we have

$$
\int_{M_{t}}|\nabla \xi|^{2} \omega^{n-1} \leq C \int_{M_{t}}\left(|\xi|+\log |t|^{-1}\right) \omega^{n-1} \leq C \log |t|^{-1} .
$$

If $E$ is a measurable subset of $M_{t}$, then we have

$$
\left|\int_{E} \partial \xi \wedge \bar{\partial} \theta \wedge \omega^{n-2}\right| \leq \int_{E}|\partial \xi| \leq C \log |t|^{-1} \sqrt{\operatorname{meas}(E)}
$$

by the Cauchy inequality.

Proof of Theorem 1.1. By Proposition 3.1, we have

$$
\begin{aligned}
& \sum_{i=0}^{n} \int_{M_{t} \cap \cup Q_{i}} \sum_{A=0}^{n}\left(\frac{X F_{t}}{\left|\nabla F_{t}\right|^{2}}\right)_{A}\left(\overline{\left.F_{t}\right)_{A}} \omega^{n-1}\right. \\
= & -\delta d-\sum_{i=0}^{n} \int_{0}^{\infty} \varphi_{i}^{\prime}(x)\left(\varphi_{i}^{\prime}(x)-1\right) d x+o(1)
\end{aligned}
$$

as $t \rightarrow 0$. We are going to prove that

$$
\int_{M_{t} \backslash_{i=0}^{n} Q_{i}} \sum_{A=0}^{n}\left(\frac{X F_{t}}{\left|\nabla F_{t}\right|^{2}}\right)_{A}\left(\overline{\left.F_{t}\right)_{A}} \omega^{n-1}=o(1)\right.
$$

as $t \rightarrow 0$. In order to see this, let's recall that we have

$$
\begin{aligned}
\int_{M_{t} \bigcup_{i=0} \cup_{Q_{i}}} \frac{\sqrt{-1}}{2 \pi} \partial \xi \wedge \bar{\partial} \theta \wedge \omega^{n-2}= & -\frac{1}{n-1} \int_{M_{t} \backslash_{i=0}^{n} Q_{i}}\left(\sum _ { A = 0 } ^ { n } ( \frac { X F _ { t } } { | \nabla F _ { t } | ^ { 2 } } ) _ { A } \left(\overline{\left.F_{t}\right)_{A}}\right.\right. \\
& \left.-\frac{\sum_{i=0}^{n} \lambda_{i}\left|\left(F_{t}\right)_{i}\right|^{2}}{\left|\nabla F_{t}\right|^{2}}+(d-1) \theta\right) \omega^{n-1}
\end{aligned}
$$


by Lemma 2.4. Since $\theta$ and the function $\frac{\sum_{i=0}^{n} \lambda_{i}\left|\left(F_{t}\right)_{i}\right|^{2}}{\left|\nabla\left(F_{t}\right)\right|^{2}}$ are bounded, we have

$$
\int_{M_{t} \backslash \underbrace{n}_{i=0} Q_{i}} \mid \sum_{A=0}^{n}\left(\frac{X F_{t}}{\left|\nabla F_{t}\right|^{2}}\right)_{A}\left(\overline{\left.F_{t}\right)_{A}} \mid \omega^{n-1} \leq \int_{M_{t} \backslash \bigcup_{i=0}^{n} Q_{i}}(|\partial \xi|+1) \omega^{n-1},\right.
$$

by (2.28). By Lemma 3.7 the righthanded side of the above equation is less than or equal to

$$
C \sqrt{\log |t|^{-1}} \sqrt{\operatorname{meas}\left(M_{t} \backslash \bigcup_{i=0}^{n} Q_{i}\right)}+\operatorname{meas}\left(M_{t} \backslash \bigcup_{i=0}^{n} Q_{i}\right) .
$$

If we can prove that there is a constant $C$ such that

$$
M_{t} \backslash \bigcup_{i=0}^{n} Q_{i} \subset \underset{i \neq j}{\cup} V_{i j}^{C t} .
$$

Then (3.22) will follow from Lemma 3.6. To see (3.23), let's consider a point $p \in M_{t} \backslash \bigcup_{i=0}^{n} Q_{i}$. Without losing generality, we assume that $p \in U_{0}$. By (3.7), we can find a $k \neq 0$ such that

$$
\left|Z_{k}\right| \leq|t|^{\sigma}\left|Z_{0}\right|
$$

for $t$ small enough. By definition, $p \notin Q_{k}$. Thus there is a $j \neq 0, k$ such that

$$
\left|Z_{j}\right| \leq|t|^{\sigma}\left|Z_{0}\right|
$$

Thus $p \in V_{j k}^{C t}$ for some constant $C$. (3.23) is proved.

Combining (3.21) and (3.22), we have

$$
\int_{M_{t}} \sum_{A=0}^{n}\left(\frac{X F_{t}}{\left|\nabla F_{t}\right|^{2}}\right)_{A}\left(\bar{F}_{t}\right)_{A} \omega^{n-1}=-\delta d-\sum_{i=0}^{n} \int_{0}^{\infty} \varphi_{i}^{\prime}(x)\left(\varphi_{i}^{\prime}(x)-1\right) d x+o(1)
$$

as $t \rightarrow 0$. Finally, since $\theta$ is a bounded function

$$
\int_{M_{t}} \theta \omega^{n-1}=\int_{M_{0}} \theta \omega^{n-1}+o(1)
$$

as $t \rightarrow 0$, where $M_{0}$ is defined as the zero set of $Z_{0}^{\alpha_{0}^{0}} \cdots Z_{n}^{\alpha_{n}^{0}}=0$ counting the multiplicity. In [5, Theorem 5.1], it is proved that

$$
\int_{M_{0}} \theta \omega^{n-1}=-\frac{\delta}{n}
$$

By (2.35), we have

$$
t \mathcal{M}^{\prime}(t)=\frac{2}{d}\left(\frac{\delta(n+1)(d-1)}{n}+\sum_{i=0}^{n} \int_{0}^{\infty} \varphi_{i}^{\prime}(x)\left(\varphi_{i}^{\prime}(x)-1\right) d x\right)+o(1)
$$

as $t \rightarrow 0$ and Theorem 1.1 is proved. 


\section{Further Discussions.}

In this section, we study the relations between the $K$-stability and the ChowMumford stability for algebraic varieties. First, we have the following

Definition 4.1. Using our notations in the first section, then for a hypersurface $M$ (not necessarily smooth) of $C P^{n}$ defined by a polynomial $F$ in (1.2), it is Chow-Mumford stable if $\lambda>0$ for any $\left(\lambda_{1}, \cdots, \lambda_{n}\right)$ with $\sum \lambda_{i}=0$, where $\lambda$ is defined in (1.3). It is Chow-Mumford unstable, if $\lambda<0$ for any $\left(\lambda_{0}, \cdots, \lambda_{n}\right)$ with $\sum \lambda_{i}=0$.

Using Theorem 1.1, we have the following

Proposition 4.1. If $M$ is $K$-stable, then $M$ is not Chow-Mumford unstable.

Proof. Let $\left(\lambda_{0}, \cdots, \lambda_{n}\right)$ be an arbitrary $(n+1)$ rational numbers with $\sum \lambda_{i}=0$. Then we can perturb $\left(\lambda_{0}, \cdots, \lambda_{n}\right)$ so that it is "generic". By Theorem 1.1 we have

$$
-\frac{\lambda(d-1)(n+1)}{n}+\sum_{i=0}^{n} \int_{0}^{\infty} \varphi_{i}^{\prime}(x)\left(\varphi_{i}^{\prime}(x)-1\right) d x \leq 0 .
$$

Since the second term above is nonnegative, we have $\lambda \geq 0$. This is a contradiction.

A well-known fact about the smooth hypersurface is that it is always Chow-Mumford stable. So the above proposition gives no new information about the stability. However, it would be interesting to generalize the notion of $K$ stability into singular varieties.

In [16], Tian defined the generalized $K$ energy on normal varieties. In the case of hypersurfaces, we can define the $K$ energy for algebraic cycles of multiplicity one. The following lemma is interesting:

Lemma 4.1. Let $M$ be a divisor of $C P^{n}$ defined by a homogeneous polynomial $F$ of multiplicity one ${ }^{1}$. Then $\mathcal{M}(t)$ in $(2.34)$ is well-defined and we call $\mathcal{M}(t)$ the generalized $K$ energy on $M$.

Remark 4.1. Clearly a normal hypersurface is defined by an irreducible polynomial. So the above result is a generalization of the result in [16] in the case of hypersurfaces. But the proof below is essentially the same as in that paper.

\footnotetext{
${ }^{1}$ A polynomial is of multiplicity one, if $\{F=0\} \cap\{\nabla F=0\}$ is of codimension at least 2 .
} 
By Lemma 2.4, Lemma 4.1 follows from the following

Lemma 4.2. Let $\xi$ be defined by Lemma 2.1. Then we have

$$
\int_{M}|\partial \xi| \omega^{n-1}<+\infty
$$

Proof. Let $M_{i}$ be an irreducible component of $M$ and let $\pi_{i}: \tilde{M}_{i} \rightarrow M_{i}$ be a smooth resolution of $M_{i}$. Then $\pi_{i}^{*}\left(e^{\xi}\right)$ is an analytic function on $\tilde{M}_{i}$. Thus we have

$$
\int_{\tilde{M}_{i}}\left|\pi_{i}^{*}(\xi)\right|^{2} \pi_{i}^{*}\left(\omega^{n-1}\right)<+\infty
$$

We also have

$$
\frac{\sqrt{-1}}{2 \pi} \partial \bar{\partial} \xi \geq-C \omega
$$

for some constant $C$ on $M_{i}$. Let $\sup \xi=C_{1}$. Then we have

$$
\int_{\tilde{M}_{i}} \frac{1}{C_{1}-\xi+1}(\sqrt{-1} \partial \bar{\partial} \xi+C \omega) \wedge \omega^{n-2} \geq 0 .
$$

Consequently we have

$$
\int_{M} \frac{1}{\left(C_{1}-\xi+1\right)^{2}}|\partial \xi|^{2} \omega^{n-1}<+\infty .
$$

Since $M$ has only finitely many components. Using the Cauchy inequality, we have

$$
\left(\int_{M}|\partial \xi| \omega^{n-1}\right)^{2} \leq \int_{M} \frac{1}{\left(C_{1}-\xi+1\right)^{2}}|\partial \xi|^{2} \omega^{n-1} \int_{M}\left(C_{1}-\xi+1\right)^{2} \omega^{n-1} .
$$

The lemma thus follows from (4.1) and (4.2).

By the above lemma, we can define $K$ stability for singular varieties. It is interesting to compare $K$ stability with Chow-Mumford stability for these singular varieties. A more interesting and nonlinear problem would be that whether the $K$ energy is proper and whether the Moser-Trudinger inequality is true for Fano hypersurfaces. The author strongly believe that they are true, regardless of the existence of Kähler-Einstein metrics.

We end up this paper by giving some observations related to the recent work of Donaldson [4]. We first setup the notations.

Let $(M, L)$ be a polarised Kähler manifold. That is, $L$ is an ample line bundle over the compact complex manifold $M$. We have the following 
setting of the metrics: let $h$ be a Hermitian metric on the line bundle $L$ with positive curvature Ric $(h)$. We use $\omega_{h}=\operatorname{Ric}(h)$ to be the Kähler metric of the manifold $X$. The pair of metrics $\left(h, \omega_{h}\right)$ induces an $L^{2}$ metric on the complex vector space $H^{0}\left(M, L^{m}\right)$, where $m$ is a large integer. Let $S_{1}, \cdots, S_{d}$ be an orthonormal basis of $H^{0}\left(M, L^{m}\right)$ under the $L^{2}$ metric. The quantity

$$
\eta_{m}=\sum_{i=1}^{d}\left\|S_{i}\right\|^{2}
$$

plays an important role in complex geometry. In particular, integrating the quantity $\eta_{m}$, we get the Riemann-Roch Theorem:

$$
\operatorname{dim} H^{0}\left(M, L^{m}\right)=\int_{M} \eta_{m} \omega^{n}=\int_{M} \operatorname{Td}(R) e^{m \omega}
$$

where $R$ is the curvature tensor of the metric $\omega$, and $\operatorname{Td}(R)$ is the Todd polynomial of $R$.

By the result of Catlin [1] and Zelditch [18] (independently), there is an asymptotic expansion

$$
\sum_{i=1}^{d}\left\|S_{i}\right\|^{2} \sim m^{n}\left(a_{0}+\frac{a_{1}}{m}+\cdots+\frac{a_{k}}{m^{k}}+\cdots\right)
$$

in the sense that

$$
\left\|\sum_{i=1}^{d}\right\| S_{i}\left\|^{2}-m^{n}\left(a_{0}+\frac{a_{1}}{m}+\cdots+\frac{a_{k}}{m^{k}}\right)\right\|_{C^{l}} \leq \frac{C(k, l, X)}{m^{k+1}},
$$

where the constant $C(k, l, X)$ depends on $k, l$ and the manifold $M$. If for some $m$, we can make the quantity $\eta_{m}$ a constant, then the manifold $M$ is Hilbert-Mumford stable [9, 19]. This result was used by Donaldson [3] in his work to prove the stability of manifolds with constant scalar curvature.

In [6], the author proved that

$$
\left\{\begin{array}{l}
a_{0}=1 \\
a_{1}=\frac{1}{2} \rho \\
a_{2}=\frac{1}{3} \Delta \rho+\frac{1}{24}\left(|R|^{2}-4|R i c|^{2}+3 \rho^{2}\right)
\end{array},\right.
$$

where $R, \operatorname{Ric}, \rho$ represent the curvature, the Ricci curvature and the scalar curvature of the Kähler metric $\omega_{h}$. The author also proved in the same paper that $a_{k}(k \in Z)$ is a universal polynomial of the curvature and its derivatives. 
Because of the above results, we can view (4.3) as a kind of local Riemann-Roch Theorem. It would be very interesting to find the general formula of $a_{k}$.

The first section of the paper [4] hints that there might be relations between the coefficients $\left\{a_{k}\right\}$ and the equivariant cohomology. The following observation of the author supports such a speculation.

We assume that $M$ is embedded to $C P^{N}$ and $L$ is the restriction of the hyperplane bundle to $M$. Let $X$ be a holomorphic vector field on $C P^{N}$ that is tangent to $X$. Let $\theta$ be the Hamiltonian function of $X$. That is,

$$
\iota(X) \omega=\frac{\sqrt{-1}}{2 \pi} \bar{\partial} \theta
$$

where $\omega$ is the Kähler metric of $M$. Let $\left(z_{1}, \cdots, z_{n}\right)$ be a local holomorphic coordinates of $M$. We define

$$
\nabla X=X_{l}^{k} \frac{\partial}{\partial z_{k}} \otimes d z_{l}
$$

where

$$
X_{l}^{k}=\frac{\partial X^{k}}{\partial z_{l}}+\Gamma_{l m}^{k} X^{m}
$$

The following identity is straightforward

$$
\iota(X) R=\frac{\sqrt{-1}}{2 \pi} \bar{\partial} \nabla X,
$$

where $R=R_{j k \bar{l}}^{i}$ is the curvature tensor. Then we have the following identities:

$$
\left\{\begin{aligned}
\int_{M} a_{0} \theta d V_{M}= & \frac{1}{(n+1) !} \int_{M} T d_{0}(R+\nabla X)(\omega+\theta)^{n+1} \\
\int_{M} a_{1} \theta d V_{M}= & -\frac{1}{(n) !} \int_{M} T d_{1}(R+\nabla X)(\omega+\theta)^{n} \\
\int_{M} a_{2} \theta d V_{M}= & -\frac{1}{(n-1) !} \int_{M} T d_{2}(R+\nabla X)(\omega+\theta)^{n-1} \\
& +\frac{1}{n !} \int_{M} T d_{1}(R+\nabla X) \Delta \theta \omega^{n}
\end{aligned}\right.
$$

where $\mathrm{Td}_{k}$ is the $k$-th homogeneous polynomial of the Todd invariant function. These identities can be verified directly. The third identity is complicated so that it should not be a coincident.

By the above observation, we have the following

Proposition 4.2. Using the notations as above, then $\int_{M} a_{k} \theta d V_{M}$ for $k=$ $0,1,2$ are independent of the choice of the Kähler metric in the fixed polarization $c_{1}(L)$. They are generalized Futaki invariants. 
Proof. We have $(\bar{\partial}-\iota(X))(\omega+\theta)=0$ and $(\bar{\partial}-\iota(X))(R+\nabla X)=0$. Thus the integrand $a_{k} \theta$ is closed under the operator $\bar{\partial}-\iota(X)$. The proposition follows from this fact.

There are several directions realted to this observation. First, it would be interesting to see that the expansion (4.3) is related to the (generalized) Futaki invariants; second it may be possible to find the general formula of $\left\{a_{k}\right\}$ in terms of equivariant cohomology; more importantly, in [8], we pointed out that $a_{k}=f$ is an elliptic equation, where $f$ is a smooth function. In particular, $a_{1}=$ const is the equation for finding Kähler metrics of constant scalar curvature. Thus prescribing $a_{k}$ for $k>1$ is interesting. We will study these questions in our subsequent papers.

\section{References.}

[1] D. Catlin. The Bergman kernel and a theorem of Tian. In Analysis and geometry in several complex variables, Trends Math., pages 1-23. Birkhüser, 1997.

[2] W. Ding and G. Tian. Kähler-Einstein metrics and the generalized Futaki invariant. Invent Math, 110:315-335, 1992.

[3] S. K. Donaldson. Scalar curvature and projective embeddings. I. J. Differential Geom., 59(3):479-522, 2001.

[4] S. K. Donaldson. Scalar curvature and stability of toric varieties. J. Differential Geom., 62(2):289-349, 2002.

[5] Z. Lu. On the Futaki invariants of complete intersections. Duke Math. J., 100(2):359-372, 1999.

[6] Z. Lu. On the lower order terms of the asymptotic expansion of TianYau-Zelditch. Amer. J. Math, 122:235-273, 2000.

[7] Z. Lu. $K$ energy and $K$ stability on hypersurfaces. DG/0108009, 2001.

[8] Z. Lu and G. Tian. The log term of Szegö kernel. preprint, 2002.

[9] H. Luo. Geometric criterion for Gieseker-Mumford stability of polarized manifolds. J. Diff. Geom, 49(3):577-599, 1998.

[10] T. Mabuchi. K-energy maps integrating Futaki invariants. Tohuku Math J., 38:245-257, 1986. 
[11] S. Paul. Geometric analysis of chow mumford stability. to appear in Advance Math.

[12] S. Paul and G. Tian. Analysis of geometric stability. preprint.

[13] D. H. Phong and J. Sturm. On asymptotics for the Mabuchi energy functional. DG/0312528.

[14] D. H. Phong and J. Sturm. Algebraic estimates, stability of local zeta functions, and uniform estimates for distribution functions. Ann. of Math. (2), 152(1):277-329, 2000.

[15] D. H. Phong and J. Sturm. The Futaki invariant and the Mabuchi energy of a complete intersection. Comm. Anal. Geom., 12:323-345, 2004 .

[16] G. Tian. The K-energy on hypersurfaces and stability. Comm. Anal. Geom, 2(2):239-265, 1994.

[17] G. Tian. Kähler-Einstein metrics with positive scalar curvature. Invent. Math, 137:1-37, 1997.

[18] S. Zelditch. Szegö Kernel and a Theorem of Tian. Internat. Math. Res. Notices, 6:317-331, 1998.

[19] S. Zhang. Heights and reductions of semi-stable varieties. Compositio Math, 104:77-105, 1996.

Department of Mathematics

University of CALIFORNiA, IRVINE

IRVINE, CA 92697

zlu@math.uci.edu

ReCeived November 6, 2003

REVISED FEBRUARY 2004. 